\title{
Propuesta de un índice sintético adelantado de desempeño fiscal para el estado de Querétaro
}

\section{Proposal for an advanced synthetic index of fiscal performance for the State of Querétaro}

Omar Bautista Hernández

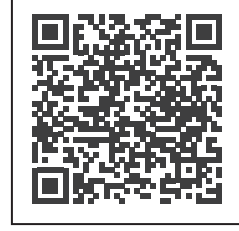

Palabras clave:

análisis estadísti-

co; análisis factorial;

análisis multivariado;

impuestos; indica-

dores económicos;

política fiscal; sistema

tributario.

Artículo de investigación

Fecha de recepción:

7 de octubre de 2021

Fecha de aprobación:

22 de noviembre de 2021

Fecha de publicación:

17 de enero de 2022

Esta publicación se encuentra bajo licencia:

Creative Commons

Reconocimiento-

NoComercial-SinObraDerivada 4.0 Internacional

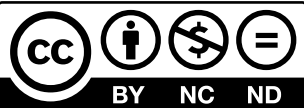

\section{Resumen}

Problemática: la presente investigación surge de la necesidad de contar con un índice como instrumento para evaluar el nivel de gestión de las finanzas públicas del estado de Querétaro. Objetivos: evaluar el desempeño fiscal del estado de Querétaro a partir de la construcción de un índice sintético adelantado de desempeño fiscal. Materiales y métodos: se realizó una revisión bibliográfica para determinar las dimensiones y los indicadores del desempeño fiscal en México. Posteriormente, se empleó la técnica de análisis de componentes principales y la media geométrica para obtener el índice sintético adelantado de desempeño fiscal. Resultados: se muestran los resultados integrales del desempeño fiscal en el estado de Querétaro para el período 2010-2019, donde resalta el resultado positivo en los últimos tres años en materia fiscal y el deterioro que presenta el índice a partir de 2019. Discusión: en el estudio se resalta el papel que tienen los gobiernos de los territorios como actores de cambio en la estructura económica y fiscal, así como la importancia en la aplicación que tienen las técnicas de estadística multivariada. Conclusiones: con los métodos de estadística multivariada se puede agrupar y sintetizar un conjunto de variables que permita explicar de forma más simple el

1 Contador Público y maestro en Impuesto, Facultad de Contaduría y Administración, Universidad Autónoma de Querétaro, México, omaruaq1@hotmail. com, ORCID: https://orcid.org/0000-0003-0027-0898 


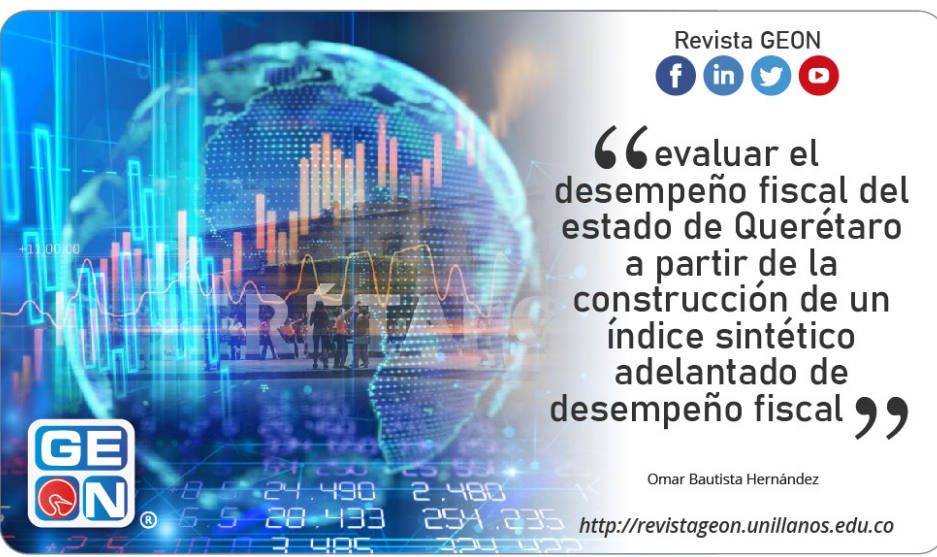

desempeño fiscal. También se estableció un criterio de clasificación según el resultado del índice sintético en: bajo, medio, alto y muy alto. Contribución/ originalidad: la contribución está en su aplicación práctica para medir y evaluar el desempeño fiscal del estado de Querétaro, la cual puede ser replicable en todos los estados de la república mexicana. Además, contribuye con métricas adicionales y al análisis del comportamiento de varios indicadores.

Palabras clave: análisis estadístico; análisis factorial; análisis multivariado; impuestos; indicadores económicos; política fiscal; sistema tributario.

Códigos JEL: E62 Política fiscal; Gasto, inversión y finanzas públicas; Tributación, H2 Tributación, subvenciones e ingresos; H2O Generalidades; H83 Administración pública.

\section{Abstract}

Problematic: The present research arises from the need of having an index as an instrument to evaluate the management level of the public finances of the State of Queretaro. Objectives: Assess the fiscal performance of the State of Queretaro, based on the construction of a symmetric forward-looking index of fiscal performance Materials and methods: A bibliographic review was performed, in order to determine the dimensions and the indicators of fiscal performance in Mexico. Afterwards, the technique for the analysis of the main components 
Cómo citar este artículo / Toreference this article:

Bautista-Hernández, O. (2022). Propuesta de un índice sintético adelantado de desempeño fiscal para el estado de Querétaro. Revista GEON (Gestión, Organizaciones Y Negocios), 9(1), e-752. https://doi. org/10.22579/23463910.752 was used, and the geometric mean to obtain the advanced summary index of fiscal performance. Results: Integral results are shown of the fiscal performance in the Sate of Queretaro for the period 2010-2019, where it outstands the positive result in the last three years in fiscal matters, and the deterioration that the index shows as of the year 2019.

Discussion: In the study, is stressed the role that the governments of the territories play as actors of change in the economic and fiscal structure, as well as the importance of applying the techniques of multivariate statistics Conclusions: With the methods of multivariate statistics, it can be grouped and synthetized a group of variables that allow to explain in a simpler manner the fiscal performance. A classification criteria was also established, according to the synthetic index, classified as low, medium, high and very high. Contribution/originality: The contribution is based on its practical application in order to measure and assess the fiscal performance of the State of Queretaro, which can be replicable in all the States of the Mexican Republic. Furthermore, it contributes with additional metrics and for the analysis of the behavior of several. Indicators.

Keywords: economic indicators; factor analysis; fiscal policy; multivariate analysis; statistical analysis; tax system; taxes.

JEL Codes E62 Fiscal Policy, H2 Taxation, Subsidies, and Revenue, H20 General, H83 Public Administration, Public Sector Accounting and Audits

\section{Introducción}

En los últimos años, la evaluación del desempeño fiscal cobra gran importancia a la hora del análisis y evaluación de la gestión de los gobiernos locales, así como del control y la administración de los recursos estatales. De igual manera, el análisis del comportamiento del desempeño fiscal permite conocer la efectividad en el manejo de dichos recursos (Madrigal-Delgado et al., 2018). En el caso particular de México, de acuerdo con lo señalado por autores como Flores Araujo y Martínez Gómez (2019), Huerta Villalobos et al. (2021), Madrigal-Delgado y Bueno-Cevada (2019) y Montiel-Morales et al. (2020), se busca fortalecer el proceso de participación 
y rendición de cuentas por medio de nuevos mecanismos de gestión pública, entre los que se encuentra el Plan Nacional de Desarrollo (PND) del período 2019-2024.

El PND es un instrumento diseñado para el aseguramiento y el fomento de la transparencia y la toma de decisiones de los gobiernos locales, el uso eficiente de los recursos y el aprovechamiento de las potencialidades de cada territorio, que se vale de instrumentos y políticas públicas con un corte transversal, las cuales están dirigidas al mejoramiento de la calidad de vida de la población (Pliego Quintana, 2014; Valencia et al., 2015; Valenzuela-Reynaga \& Hinojosa-Cruz, 2017).

En este sentido, uno de los principales problemas que afectan a las finanzas públicas de los estados y municipios mexicanos es la baja captación de ingresos propios, entre ellos los ingresos fiscales (tales como impuestos, derechos, productos y aprovechamientos), pues todavía persisten algunas insuficiencias en el sistema de recaudación fiscal, los registros de catastro son obsoletos o porque no se quiere producir insatisfacción de los gobernados, lo que provocaría una baja popularidad del gobernante local. Lo anterior indica que los ingresos fiscales se encuentran limitados por el costo político, lo que lleva a un déficit para cubrir los gastos públicos dentro del gobierno local (López et al., 2020; López Pérez \& Vence, 2021; Llamas Rembao et al., 2020; Mendoza Velázquez \& Rubio García, 2020). Además, como señalan Ibarra Salazar et al. (2013) y Madrigal-Delgado et al.
(2018), esto provoca que los gobiernos locales dependan de la transferencia del gobierno federal y de las transferencias de los deudores para asegurar su capacidad financiera, ya que cualquier desajuste les trae serias afectaciones económicas.

Con la actual pandemia por el SARSCoV-2 se ha incrementado el adeudo al erario por parte de las empresas y personas particulares. Según las cifras de la Secretaría de Hacienda y Crédito Público (SHCP) en su último informe, este adeudo pasó de ser de más de 782.000 millones de pesos durante el tercer trimestre de 2020 a más de 1 billón de pesos con respecto al mismo período en 2021. De igual manera, se ha debilitado la recaudación de los ingresos tributarios en los municipios, donde se han presentado altos índices de pereza fiscal. En este aspecto, al cierre de 2020, según datos aportados por el Instituto Nacional de Estadística y Geografía (INEGI), los estados de Ciudad de México, Baja California, Jalisco, Nuevo León y Tamaulipas son los que presentan valores de recaudación de impuesto por encima del promedio nacional de \$4100 millones de pesos mexicanos. Por su parte, los estados de Aguascalientes, Baja California Sur, Guerrero, Nayarit y Tlaxcala son las entidades federativas que tienen el nivel más bajo de recaudación de impuestos y los estados con mayor morosidad. Sin embargo, existen limitantes para las entidades federativas puesto que la recaudación de impuestos es de aproximadamente del 5,0\%. Por su parte, el gobierno federal recauda más de un 92 \% (Moreno-Brid et al., 2019). 
Por otro lado, el estado de Querétaro se encuentra ubicado entre los primeros lugares del ranking de transparencia fiscal en México, con base en el índice de transparencia y disponibilidad de la información fiscal 2021 (ITDIF), que le otorga un valor de 98,68 de 100 puntos que tiene en cuenta dicha evaluación. Ahora bien, el estado, pese a contar con una evaluación satisfactoria en materia de transparencia fiscal, que en la recaudación de impuestos ha tenido una tendencia al incremento en los últimos años (figura 1), en el período comprendido entre el 2012 y el 2019 no ha superado la media nacional de recaudación de impuestos.

Por tal motivo existe la necesidad de contar con un índice que sirva de complemento al ITDIF y como instrumento para evaluar el nivel gestión de las finanzas públicas del estado. En la literatura, autores como Cano Ortega (2019), Carvajal y López-Camargo (2020), Hernández Ocampo (2017), Hernández García et al. (2020), Leila (2021), Madrigal-Delgado et al. (2018), Pliego Quintana (2014), OCDE (2009), Sánchez-Torres (2008), Sánchez et al. (2016) y Urrea et al. (2020) señalan la importancia de contar con un índice o indicador que permita la evaluación y el análisis de manera integral del desempeño en materia de gestión fiscal.

Por tanto, la presente investigación tiene el objetivo de evaluar el desempeño fiscal del estado de Querétaro a partir de la construcción de un índice sintético adelantado de desempeño fiscal. Para ello se utilizó la técnica de estadística multivariada-análisis de componentes principales para la creación de un índice que facilite la

Figura 1. Recaudación de impuestos del estado de Querétaro, 2012-2019.

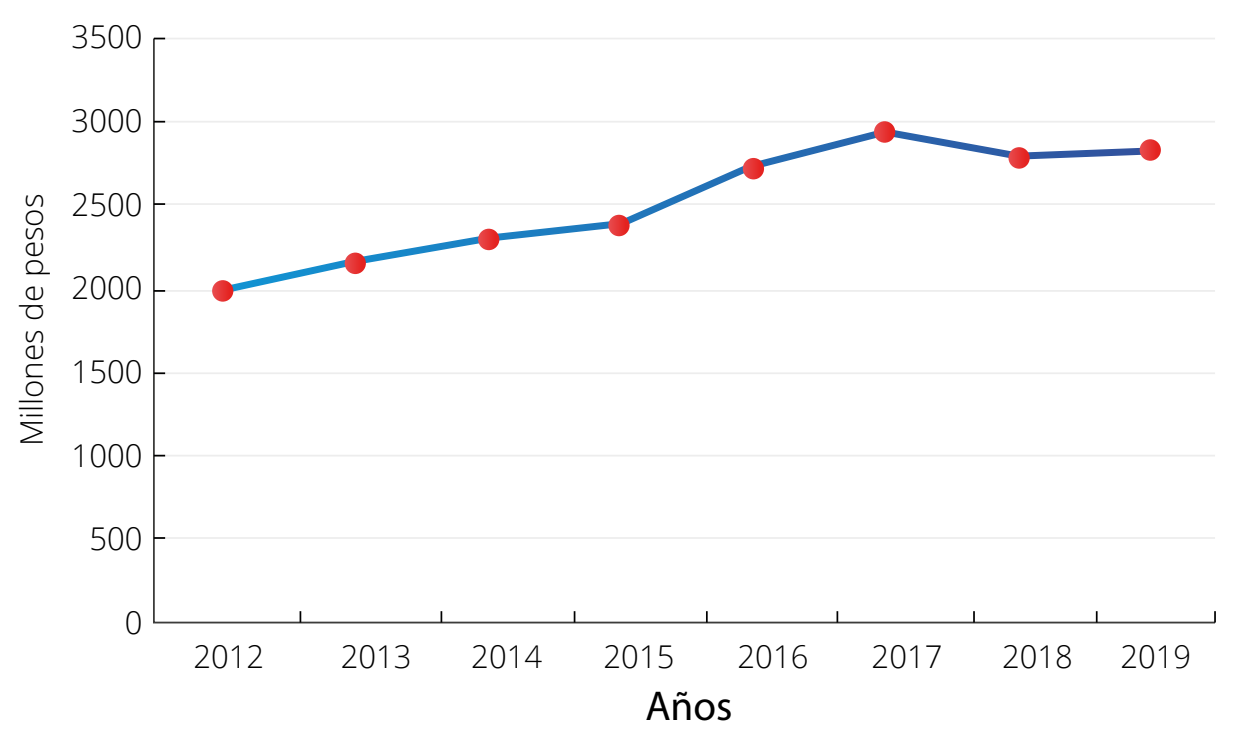

Fuente: elaboración propia a partir de los datos del INEGI (2020). 
evaluación integral del desempeño fiscal en el estado de Querétaro.

La presente investigación se encuentra organizada de la siguiente manera: un primer acápite que se compone del marco teórico, donde se aborda la conceptualización del desempeño fiscal. Posteriormente, los principales elementos y definiciones de índices sintéticos; y finalmente, la utilización de los índices sintéticos en la evaluación del desempeño fiscal. Un segundo acápite, donde se presentan los elementos metodológicos para la construcción del índice sintético adelantado de desempeño fiscal. Un tercer acápite, donde se presentan los principales resultados de la investigación. Un cuarto acápite, donde se realiza una breve discusión de los resultados; y, para finalizar, las conclusiones de la investigación.

\section{Contexto teórico}

\section{Desempeño fiscal}

Para hablar del desempeño fiscal de un país, estado o municipio, es inevitable establecer una conceptualización que se relacione con el proceso de recaudación fiscal. El desempeño corresponde a los resultados de carácter individual y colectivo dentro de un país, estado o región y la armonización del proceso de gestión fiscal en la consecución de las metas y objetivos del territorio (Lami et al., 2021; Sánchez León, 2021; Reza-Paocarina et al., 2021; Wang et al., 2021). De modo que, en el caso particular de México, el desempeño fiscal hace referencia al instrumento para reducir la evasión fiscal y la deuda pública, al igual que garantizar la correcta gestión de los recursos financieros del estado que permita bridar mejores servicios de calidad en los sectores de educación, salud, seguridad y justicia, además de combatir la pobreza e impulsar el proceso de cambio estructural de la economía y el crecimiento económico.

En este marco, autores como El Anshasy y Katsaiti (2013), Eyraud y Lusinyan (2013), Silva et al. (2020) y Cochrane (2021) señalan que el desempeño fiscal es un sistema complejo de relaciones entre los gobernantes y gobernados; de ahí lo importante de contar con indicadores fiscales que permitan la evaluación del desempeño de los gobiernos locales y, a su vez, conocer el nivel de cumplimiento de sus metas para alcanzar la armonía con la política fiscal establecida por país. En otras palabras, medir el desempeño fiscal en el estado es el camino para lograr la transparencia fiscal y la rendición de cuenta de los gobernadores de cada estado en cuanto a la correcta administración de los recursos financieros, lo cual se interpreta como finanzas sanas y sostenibles, incremento de los niveles de ahorros en el estado, nivel de endeudamiento sostenible, aumento de las inversiones y gastos públicos que pueden ser pagados con recursos propios.

\section{Conceptualización de los índices sintéticos}

En la actualidad, los índices sintéticos se han convertido en uno de 
los instrumentos más importantes a la hora de realizar mediciones analíticas en diversos campos de la ciencia. Es así como cada vez tienen mayor aceptación en el proceso de toma de decisiones y en la presentación de la información, por lo cual resulta importante establecer su conceptualización.

En este sentido, para los autores Mondéjar-Jiménez y Vargas-Vargas (2008) se denomina índice sintético a la combinación lineal de un conjunto de indicadores individuales, los cuales pueden ser llamados como indicadores componentes (p. 567). Por su parte, Blancas Peral et al. (2006) define al índice sintético como la agregación matemática de diferentes indicadores o componentes de un concepto que se desea analizar y que proporciona una valoración del objeto de estudio a una escala.

Por consiguiente, son muchas las aplicaciones que tiene el uso de los índices sintéticos, pues fácilmente puede resumirse un grupo de indicadores en un índice, lo que facilita la interpretación de la información y el análisis de las variables por parte de los usuarios, además de realizar evaluaciones de sucesos o constructos complejos rápidamente. Por ello, el principal objetivo de la construcción de los índices sintéticos es facilitar el proceso de toma de decisiones. Desde una perspectiva metodológica, la construcción de índices sintéticos lleva al establecimiento de un procedimiento matemático para lograr la combinación lineal de indicadores individuales para realizar la medición de conceptos que cuentan con múltiples dimensiones y que pueden ser representados en índice con una sola unidad de medida.

\section{Índices sintéticos para la evaluación del desempeño fiscal}

Para realizar una evaluación en materia de desempeño fiscal, es necesario efectuar un estudio y revisión bibliográfica para establecer las regularidades en las metodologías empleadas por diversos autores. Entre las técnicas de mayor aceptación en la literatura se encuentran los modelos de lógica difusa, el análisis factorial por componentes principales, las técnicas de clasificación análisis discriminante y el análisis de clúster. En cuanto a los estudios, está el desarrollado por Molinos-Senante et al. (2021), quienes utilizan el método de componentes principales para la construcción de un índice sintético para la medición de la calidad del servicio de las empresas de agua.

Por su parte, Tinto Arandes et al. (2021) realizaron un trabajo donde aplicaron un método multivalente para la creación de un índice sintético global del desarrollo sostenible y utilizaron un modelo de lógica difusa para recoger la incertidumbre y establecer mediciones de un conjunto de variables.

En el caso de nuestro continente, destacan los estudios de Gómez Giraldo et al. (2013), los cuales realizan un análisis descriptivo mediante un índice sintético de desempeño fiscal de las entidades territoriales, mien- 
tras que Andalle (2020) lleva a cabo un análisis cuantitativo en el que aplica el método de análisis envolvente de datos (DEA) para la evaluación del desempeño de los territorios con la construcción de indicadores sintéticos para analizar las dimensiones del desempeño fiscal en los territorios.

En el caso de México, se encuentran los trabajos realizados por Madrigal-Delgado et al. (2018) y Madrigal-Delgado y Bueno-Cevada (2019), donde evalúan el desempeño fiscal con la utilización de la técnica de análisis de componentes principales (ACP) para la conformación de un indicador sintético a partir de un grupo de variables que permiten realizar una evaluación de los gobiernos locales en materia fiscal.

En este sentido, el método de ACP destaca entre los demás métodos, pues su objetivo fundamental es la transformación de un grupo de variables o indicadores simples en un nuevo conjunto, conocido como componentes principales, que cumplen con la condición de estar interrelacionados y que pueden tener un orden específico con base en la información que se encuentra implícita en ellos (Dorado Corona et al., 2021; Duque et al., 2021; Durán Romero, 2000; Mondéjar-jiménez et al., 2009; Mondéjar-jiménez \& Vargas-Vargas, 2008; Tinto Arandes et al., 2021).

Según Lacaze et al. (2021), para dar soluciones a problemas que cuentan con muchas variables, es necesaria la utilización de métodos de análisis multivariantes como instrumentos de vital importancia en la toma de decisiones en múltiples ramas de la ciencia. A partir de dichas técnicas se consigue eliminar la información poco relevante o repetitiva, de manera tal que pueda identificarse la información más relevante y definirse las variables más importantes dentro del objeto de estudio. Lo anterior se logra precisamente con la utilización de la técnica estadística ACP a partir de la reducción de dimensiones y variables, de forma tal que el índice resultante explique de la mejor manera las variables originales y muestre sus cambios en un tiempo determinado.

El ACP también es muy utilizado para fraccionar unidades muestrales en subdivisiones según su grado de similitud. De igual manera, es una herramienta útil para realizar la validación de hipótesis determinadas en una investigación e identificar aquellos valores anormales dentro de los datos del estudio. Finalmente, León González et al. (2008) afirman que el ACP permite establecer las posibles dimensiones o factores dentro de la conceptualización de un objeto de estudio y posteriormente compararlo con el análisis de clúster para su validación.

\section{Materiales y métodos}

En la literatura se proponen diversas metodologías para la obtención de índices sintéticos, aunque autores como Malvicino et al. (2020), Valencia et al. (2015), Vásquez et al. (2000), Rao (2021) y Zhang y Hussain (2021) señalan que la metodología que debe emplearse se resume en cinco pasos, que es también la empleada en esta 
investigación para la construcción del índice sintético adelantado de desempeño fiscal. A continuación, se explican cada uno de los pasos.

\section{Construir la base de datos que deben utilizarse}

Para la construcción del índice sintético se utilizaron los datos del año 2010 al 2019 que ofrece el Instituto Nacional de Estadística y Geografía (INEGI) en su sección Estadística de finanzas públicas estatales y municipales. Para la construcción de los indicadores que se utilizaron para el índice sintético adelantado de desempeño fiscal se estructuró una base de datos con los indicadores originales y posteriormente se usaron las fórmulas de cálculo de cada indicador. En total se consideraron doce indicadores y cinco dimensiones que se detallan más adelante.

\section{Determinar las dimensiones que componen el desempeño fiscal}

A partir de la revisión de la literatura de autores como León González et al. (2008), Madrigal-Delgado et al. (2018)y Ávila Rodríguez y Solano-Pérez (2018), fueron definidas cinco dimensiones que se consideraron determinantes para la evaluación del desempeño fiscal, a saber: 1) capacidad financiera y tributaria, 2) generación de recursos propios, 3) capacidad de inversión, 4) capacidad de ahorro, 5) deuda.

\section{Selección de los indicadores}

Una vez que fueron definidas las dimensiones que mejor se ajustan al desempeño fiscal en México, se procedió a definir los indicadores para cada una de las dimensiones propuestas teniendo en cuenta la información estadística disponible en el INEGl; se seleccionaron un total de nueve indicadores. En la tabla 1 se presenta la dimensión, la fórmula de cálculo, la descripción general y el objetivo de cada indicador propuesto.

\section{Obtención de las puntuaciones del índice sintético adelantado de desempeño fiscal}

En este paso se aplicó la técnica de estadística multivariada-análisis de componentes principales para obtener las puntaciones del índice sintético adelantado de desempeño fiscal, debido a su facilidad para reducir un conjunto de indicadores a un índice que explique de mejor manera el desempeño fiscal y logrando el número óptimo de dimensiones que expliquen mejor la información contenida en los datos. Por tal motivo, Tejero et al. (2011), Navarro et al. (2020) y Lacaze et al. (2021) señalan que el primer paso y uno de los más importantes para desarrollar el método es analizar el determinante de la matriz de correlaciones y si sus valores son cercanos a cero, aunque no deban ser iguales a cero, pues no se podría aplicar dicho método. Después debe evaluarse la medida de Kaiser-Meyer-Olkin (KMO) y la prueba de esfericidad de Bartlett. En el caso de la primera, Navarro et al. (2020) indican que si el valor de KMO es menor que 0,5, no es adecuado aplicar el método ya que las correlaciones entre las variables no pueden ser explicadas; entre 
Tabla 1. Indicadores para el cálculo del índice sintético adelantado de desempeño fiscal

\begin{tabular}{|c|c|c|}
\hline $\begin{array}{l}\text { Dimensio- } \\
\text { nes }\end{array}$ & Indicadores & Objetivo del indicador \\
\hline \multirow{2}{*}{$\begin{array}{l}\text { Capacidad } \\
\text { financiera y } \\
\text { tributaria }\end{array}$} & Dependencia $=\frac{\text { Tranferencias }}{\text { Totaldeingresos }}$ & $\begin{array}{l}\text { Nivel de dependencia del estado } \\
\text { con respecto a las transferencias } \\
\text { recibidas. }\end{array}$ \\
\hline & Autonomía financiera $=\frac{\text { Ingresos propios }}{\text { Ingresostotales }}$ & $\begin{array}{l}\text { Nivel de autonomía del estado res- } \\
\text { pecto a otras fuentes de financia- } \\
\text { miento. }\end{array}$ \\
\hline \multirow{3}{*}{$\begin{array}{l}\text { Generación } \\
\text { de recursos } \\
\text { propios }\end{array}$} & Generaciónrecursos propios $=\frac{\text { Ingresostributarios }}{\text { Total deingresos }}$ & $\begin{array}{l}\text { Capacidad del estado de generar } \\
\text { recursos complementarios a las } \\
\text { transferencias. }\end{array}$ \\
\hline & \multirow{2}{*}{ Capacidad de servicios $=\frac{\text { Impuestos }}{\text { Servicios personales }}$} & \multirow{2}{*}{$\begin{array}{l}\text { Capacidad del estado para cubrir } \\
\text { los gastos de servicios personales } \\
\text { con sus propias contribuciones. }\end{array}$} \\
\hline & & \\
\hline \multirow{3}{*}{$\begin{array}{l}\text { Capacidad } \\
\text { de inver- } \\
\text { sión }\end{array}$} & Inversión & \multirow{2}{*}{$\begin{array}{l}\text { Cuantifica la magnitud de la inver- } \\
\text { sión del territorio con respecto al } \\
\text { total de gastos. }\end{array}$} \\
\hline & $\overline{\text { Total de gastos }}$ & \\
\hline & Realización de la inversión $=\frac{\text { Gasto de capital }}{\text { Otrosingresos }}$ & $\begin{array}{l}\text { Capacidad del estado para cubrir } \\
\text { sus gastos de capital con otros } \\
\text { ingresos. }\end{array}$ \\
\hline $\begin{array}{l}\text { Capacidad } \\
\text { de ahorro }\end{array}$ & Capacidad de ahorro $=\frac{\text { Ahorro }}{\text { Total deingresos }}$ & $\begin{array}{l}\text { Mide el nivel en el que se liberan } \\
\text { los excedentes para financiar la } \\
\text { inversión. }\end{array}$ \\
\hline \multirow{2}{*}{ Deuda } & Sostenibilidad de la deuda $=\frac{\text { Deuda }}{\text { Total deingresos }}$ & $\begin{array}{l}\text { Mide la capacidad del estado para } \\
\text { respaldar el saldo de la deuda con } \\
\text { sus ingresos. }\end{array}$ \\
\hline & Gasto discrepcional $=\frac{\text { Servicios personales }}{\text { Total de gasto }}$ & $\begin{array}{l}\text { Muestra el porcentaje del gasto } \\
\text { corriente dedicado a servicios per- } \\
\text { sonales. }\end{array}$ \\
\hline
\end{tabular}

Fuentes: elaboración propia con base en Madrigal-Delgado et al. (2018);Gómez Giraldo et al. (2013); INEGI (2020)

0,5 y 1, el método puede ser aplicado, con especial énfasis en aquellos valores por encima de 0,8. En cuanto a la prueba de esfericidad de Bartle$\mathrm{tt}$, se compara el valor de $p$ con $\mathrm{a}, \mathrm{y}$ si el primero es menor, los datos se ajustan bien a la técnica. A continuación, se realiza el análisis de la tabla de comunalidades, varianza total explicada y la matriz de componentes. Las comunalidades muestran cómo el indicador resultante explica las variables originales; a su vez, en la tabla de varianza total explicada se muestra el porciento de información que se recoge según la cantidad de factores, y en la matriz de componentes se observa el nivel en que las variables originales explican al indicador resultante (Montenegro, 2021; Morales \& Bayona, 2020; Silva-Treviño et al., 2021). Por último, se analiza la matriz de coeficientes rotados, de donde se obtienen las puntuaciones de los índices sintético parciales que conforman el índice sintético adelantado de desempeño fiscal, con el uso del método Varimax, que reduce la cantidad de variables 
con saturaciones elevadas dentro de cada factor (Navarro et al., 2020).

\section{Construcción del índice sintético adelantado de desempeño fiscal}

Una vez que se obtuvo el número óptimo de las dimensiones o factores, se utilizó la media geométrica para el resultado del índice sintético adelantado de desempeño fiscal:

ISADF $=\sqrt[n]{D_{1} \star D_{2} \star D_{3}{ }^{*} \ldots D_{n}}(1)$

donde ISADF es índice sintético adelantado de desempeño fiscal y $D$ es las dimensiones desde 1 hasta $n$.

\section{Resultados}

Los resultados obtenidos en el trabajo de investigación se estructuran de la siguiente manera: análisis de la matriz de correlación y su determinante; prueba de KMO y prueba de esfericidad de Bartlett; análisis de las comunalidades; análisis de la varianza total explicada y la matriz de componentes rotados. A continuación, se realiza el cálculo correspondiente al índice sintético y se analiza su comportamiento en el período 2010-2019 para su validación.

En cuanto a la matriz de correlación, la mayor parte de las variables presentaron valores superiores a 0,60 y el determinante de la matriz tuvo un valor de 8,30×10-7, lo que indica un valor bien cercano a cero; por tanto, es factible aplicar el método de componentes principales. Para fortalecer el análisis anterior, es necesario validarlo a través de las pruebas de KMO y de Bartlett. En la tabla 2 se muestra que el valor de la medida de adecuación muestral es superior a 0,50, lo que indica la factibilidad del método aplicado y que el valor de $p$ es menor que un nivel de significación del 0,05, por lo cual la prueba de esfericidad de Bartlett es significativa para el estudio.

Tabla 2. Prueba des KMO y de Bartlett

\begin{tabular}{llr}
\multicolumn{2}{c|}{$\begin{array}{c}\text { Medida Kaiser-Meyer-Olkin } \\
\text { de adecuación de muestreo }\end{array}$} & $\mathbf{0 , 5 3 7}$ \\
$\begin{array}{llr}\text { Prueba de } \\
\text { esfericidad de } \\
\text { Bartlett }\end{array}$ & Aprox. Chi-cuadrado & 72,341 \\
\cline { 2 - 3 } & gl & 36 \\
\cline { 2 - 3 } & Sig. & 0,000 \\
\hline
\end{tabular}

Fuentes: salida del SPSS 24.0, con base en los datos del INEGI (2020).

De acuerdo con la información obtenida, en la tabla de comunalidades (tabla 3) se muestran los altos índices para cada una de las variables. La proporción de la varianza que puede ser explicada para cada variable fue superior a los 0,70, donde se destacan las variables capacidad de servicio, magnitud de la inversión, dependencia y autonomía financiera con valores por encima de 0,92 una vez realizada la extracción. Es decir, las variables originales quedan explicadas muy bien en el indicador resultante.

Se utilizó el método de extracción Varimax del análisis de componentes principales porque redistribuye la varianza a todos los componentes en la matriz de cargas factoriales. Lo anterior permite obtener resultados más fáciles de interpretar e identificar los factores que pertenecen a cada componente, eliminando datos confusos en la matriz de componentes rotados. Por su parte, en la tabla de varianza total explicada (tabla 4) se muestran 
los autovalores de los indicadores originales seleccionados, mientras que en la parte derecha puede observarse que las sumas de rotación de cargas al cuadrado muestran que el número óptimo de dimensiones o actores sería 3, los cuales consiguen explicar el 88,94 \% de la varianza de los datos originales.

Tabla 3. Comunalidades

\begin{tabular}{lcc} 
& Inicial & Extracción \\
\hline Dependencia & 1,000 & 0,934 \\
\hline Autonomía financiera & 1,000 & 0,925 \\
\hline Gestión de recursos propios & 1,000 & 0,874 \\
\hline Capacidad de servicio & 1,000 & 0,964 \\
\hline Magnitud de la inversión & 1,000 & 0,936 \\
\hline Capacidad de ahorro & 1,000 & 0,889 \\
\hline Gasto discrepcional & 1,000 & 0,874 \\
\hline Sostenibilidad de la deuda & 1,000 & 0,743 \\
\hline Realización de la inversión & 1,000 & 0,867 \\
\hline Método de extracción: análisis de & \\
\multicolumn{2}{l}{ componentes principales. } & \\
\hline
\end{tabular}

Fuentes: salida del SPSS 24.0, con base en los datos del INEGI (2020).

En el caso de la tabla de componentes rotados (tabla 5), puede observarse el nivel de asociación que existe entre las variables objeto del estudio. A partir de los resultados obtenidos, puede establecerse la interrelación entre aquellas variables que presenten un alto grado de correlación entre ellas.

Como se observa en el análisis, una vez aplicado el método de extracción Varimax, las variables pueden agruparse en tres dimensiones. En la primera se encuentran relacionadas las variables autonomía financiera (AutFin), gestión de recursos propios (GRprop), capacidad de ahorro (Caho) y sostenibilidad de la deuda (Sdeu), las cuales pueden agruparse en un índice, que se denominará índice parcial de capacidad y sostenibilidad (IPCS). En el segundo índice se agruparían las variables capacidad de servicio (Cserv), gasto discrepcional (Gdiscrep) y realización de la inversión (Rinv); este se llamará índice parcial de inversión (IPInv). En el tercer índice se agruparían las variables dependencia (Dep) y magnitud de la inversión (Minv); se denominará índice parcial de dependencia (IPD).

Tabla 4. Varianza total explicada

\begin{tabular}{|c|c|c|c|c|c|c|c|c|c|}
\hline \multirow{2}{*}{ Componente } & \multicolumn{3}{|c|}{ Autovalores iniciales } & \multicolumn{3}{|c|}{$\begin{array}{l}\text { Sumas de extracción } \\
\text { de cargas al cuadrado }\end{array}$} & \multicolumn{3}{|c|}{$\begin{array}{l}\text { Sumas de rotación } \\
\text { de cargas al cuadrado }\end{array}$} \\
\hline & Total & $\begin{array}{c}\% \text { de } \\
\text { varianza }\end{array}$ & $\begin{array}{c}\% \\
\text { acumulado }\end{array}$ & Total & $\begin{array}{c}\% \text { de } \\
\text { varianza }\end{array}$ & $\begin{array}{c}\% \\
\text { acumulado }\end{array}$ & Total & $\begin{array}{c}\% \text { de } \\
\text { varianza }\end{array}$ & $\begin{array}{c}\% \\
\text { acumulado }\end{array}$ \\
\hline 1 & 4,493 & 49,923 & 49,923 & 4,493 & 49,923 & 49,923 & 3,578 & 39,754 & 39,754 \\
\hline 2 & 1,878 & 20,864 & 70,788 & 1,878 & 20,864 & 70,788 & 2,507 & 27,859 & 67,613 \\
\hline 3 & 1,634 & 18,153 & 88,941 & 1,634 & 18,153 & 88,941 & 1,920 & 21,328 & 88,941 \\
\hline 4 & 0,511 & 5,682 & 94,623 & & & & & & \\
\hline 5 & 0,315 & 3,503 & 98,126 & & & & & & \\
\hline 6 & 0,118 & 1,311 & 99,436 & & & & & & \\
\hline 7 & 0,030 & 0,332 & 99,768 & & & & & & \\
\hline 8 & 0,012 & 0,136 & 99,904 & & & & & & \\
\hline 9 & 0,009 & 0,096 & 100,000 & & & & & & \\
\hline
\end{tabular}

Fuentes: salida del SPSS 24.0, con base en los datos del INEGI (2020). 
Tabla 5. Matriz de componentes rotados

\begin{tabular}{|c|c|c|c|}
\hline & \multicolumn{3}{|c|}{ Componente } \\
\hline & 1 & 2 & 3 \\
\hline Dependencia & 0,015 & 0,187 & 0,948 \\
\hline Autonomía financiera & 0,939 & 0,095 & $-0,185$ \\
\hline $\begin{array}{l}\text { Gestión de recursos } \\
\text { propios }\end{array}$ & 0,925 & 0,051 & 0,126 \\
\hline $\begin{array}{l}\text { Capacidad de } \\
\text { servicio }\end{array}$ & 0,202 & 0,960 & 0,038 \\
\hline $\begin{array}{l}\text { Magnitud de la } \\
\text { inversión }\end{array}$ & $-0,080$ & 0,088 & $-0,960$ \\
\hline Capacidad de ahorro & 0,922 & 0,186 & 0,065 \\
\hline Gasto discrepcional & $-0,615$ & $-0,697$ & $-0,101$ \\
\hline $\begin{array}{l}\text { Sostenibilidad de la } \\
\text { deuda }\end{array}$ & 0,749 & 0,386 & 0,181 \\
\hline $\begin{array}{l}\text { Realización de la } \\
\text { inversión }\end{array}$ & 0,068 & 0,928 & 0,018 \\
\hline \multicolumn{4}{|c|}{$\begin{array}{l}\text { Método de extracción: análisis de } \\
\text { componentes principales. } \\
\text { Método de rotación: Varimax con } \\
\text { normalización Kaiser. }\end{array}$} \\
\hline
\end{tabular}

Fuentes: salida del SPSS 24.0, con base en los datos del INEGI (2020).

Finalmente, se analiza la tabla 6 para obtener los valores de las cargas factoriales de cada variable de los índices parciales, las cuales permiten expresar cada índice parcial como combinación lineal de las variables que tienen un alto grado de correlación entre ellas.

Dado lo anterior, que cada índice parcial se expresa a través de las siguientes ecuaciones:

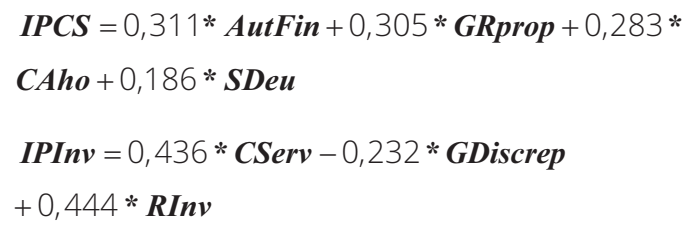

Tabla 6. Matriz de coeficiente para las puntuaciones

\begin{tabular}{|c|c|c|c|}
\hline & \multicolumn{3}{|c|}{ Componente } \\
\hline & 1 & 2 & 3 \\
\hline Dependencia & $-0,053$ & 0,045 & 0,496 \\
\hline Autonomía financiera & 0,311 & $-0,106$ & $-0,129$ \\
\hline $\begin{array}{l}\text { Gestión de recursos } \\
\text { propios }\end{array}$ & 0,305 & $-0,140$ & 0,039 \\
\hline Capacidad de servicio & $-0,097$ & 0,436 & $-0,030$ \\
\hline $\begin{array}{l}\text { Magnitud de la inver- } \\
\text { sión }\end{array}$ & $-0,015$ & 0,102 & $-0,513$ \\
\hline Capacidad de ahorro & 0,283 & $-0,070$ & 0,000 \\
\hline Gasto discrepcional & $-0,088$ & $-0,232$ & $-0,004$ \\
\hline $\begin{array}{l}\text { Sostenibilidad de la } \\
\text { deuda }\end{array}$ & 0,186 & 0,053 & 0,057 \\
\hline $\begin{array}{l}\text { Realización de la } \\
\text { inversión }\end{array}$ & $-0,137$ & 0,444 & $-0,036$ \\
\hline $\begin{array}{l}\text { Método de extracción } \\
\text { componentes principa } \\
\text { Método de rotación: } \\
\text { normalización Kaiser. }\end{array}$ & $\begin{array}{l}\text { análisis } \\
\text { es. } \\
\text { arimax }\end{array}$ & & \\
\hline
\end{tabular}

Fuentes: salida del SPSS 24.0, con base en los datos del INEGI (2020).

El índice sintético adelantado de desempeño fiscal se determinaría a partir de la siguiente fórmula:

\section{$I S A D F=\sqrt[3]{I P C S * I P I n v * I P D}$}

Se procedió con el cálculo del índice sintético adelantado de desempeño fiscal para el período 2010-2019 con los datos ofrecidos por el INEGI y se obtuvieron los siguientes resultados (tabla 7).

Por último, con el índice sintético adelantado de desempeño fiscal se procedió a establecer la clasificación según los valores en bajo, medio, alto y muy alto (tabla 8). 
Tabla 7. Valores del índice sintético adelantado de desempeño fiscal 2010-2019

\begin{tabular}{l|l} 
Año & ISADF \\
\hline 2010 & 0,1562 \\
\hline 2011 & 1,5188 \\
\hline 2012 & 0,2217 \\
\hline 2013 & 0,5123 \\
\hline 2014 & 2,1527 \\
\hline 2015 & 0,2099 \\
\hline 2016 & 1,4242 \\
\hline 2017 & 2,5250 \\
\hline 2018 & 2,9883 \\
2019 & 0,2727 \\
\hline
\end{tabular}

Fuentes: propia del autor, con datos del INEGI.

Tabla 8. Clasificación del índice sintético adelantado de desempeño fiscal

\begin{tabular}{c|c} 
ISADF & Clasificación \\
$>0,805$ & Muy alto \\
\hline $0,711-0,804$ & Alto \\
\hline $0,536-0,710$ & Medio \\
\hline$<0,535$ & Bajo \\
\hline
\end{tabular}

Fuente: elaboración propia del autor.

Como puede observarse en los resultados de la tabla 7, en los años 2010, 2012, 2013, 2015 y 2019 el estado presentó un desempeño fiscal bajo; cabe destacar que en 2019, con el comienzo de la actual pandemia, se vieron afectados muchos negocios y en este sentido disminuyeron algunos indicadores de desempeño fiscal, así como la recaudación de los impuestos, lo que indica la alta dependencia de las transferencias que tenía el estado en dichos años. Lo anterior provocaba el incumplimiento de los gastos corrientes porque su capacidad tributaria era muy baja para poder cubrir dichos gastos. Por su parte, en el período 2016-2018, el estado venía desempeñando un buen trabajo en materia fiscal, con finanzas sanas que le permitían cubrir más de un $80 \%$ sus gastos corrientes; es decir, mayor capacidad financiera y de inversión.

\section{Discusión}

En la presente investigación se evidencia que un uso correcto y sostenible de los recursos fiscales, además de potenciar la inversión, permite generar riquezas en el territorio, que pueden ser utilizadas para la creación de nuevas fuentes de empleos, potenciar diversos sectores de la economía y mejorar la calidad de vida de la población a través de proyectos sociales. Dicho planteamiento confirma lo expresado por Carvajal y López (2020), quienes indican que existe una estrecha relación entre el desempeño fiscal y la correcta gestión de los recursos económicos por parte de los gobiernos locales.

Por otra parte, Madrigal-Delgado et al. (2018) en su estudio identifican que dentro de los estados pueden existir unas marcadas diferencias en el desempeño fiscal. Aquellos que muestren un valor muy alto del índice es porque tienen una mejor estrategia de recaudación, lo cual posibilita una mayor sostenibilidad financiera y poder cubrir la mayor parte de sus gastos públicos. A su vez, tienen un menor grado de dependencia de las transferencias realizadas por el gobierno federal. Es decir, el desempeño en materia fiscal a nivel estatal tiene una relación directa con la gestión recaudatoria, los gastos públicos 
y la sostenibilidad financiera e inversa con la dependencia a las transferencias del gobierno federal.

En este orden de ideas, queda clara la relevancia que tiene para el estado de Querétaro la implementación de políticas fiscales que permitan finanzas limpias y que se busquen nuevas fuentes de autofinanciamiento con el objetivo de una mejor gestión de los recursos locales, lo cual tendría un impacto dentro de la producción, la capacidad de ahorro y el ingreso territorial que son evaluados en el ISADF. En este contexto, Ramírez Rodríguez et al. (2017) concluyen que el desempeño fiscal no depende solamente de la recaudación fiscal y la capacidad financiera, que no son los únicos determinantes en los ingresos estatales en México, sino que existe otro grupo de variables externas e internas para el incremento de los ingresos propio en los territorios y que a su vez explican el desempeño fiscal.

Por último, debe señalarse el papel que desempeñan los gobiernos de los territorios como actores de cambio en la estructura económica y fiscal. Es así como en el estudio realizado por Mendoza Velázquez (2010), con el apoyo de técnicas de estadística multivariada para estudiar la situación de las finanzas públicas estatales en México, se estima que la dinámica de las finanzas públicas de los estados en México puede ser explicada de manera confiablemente a partir de seis factores que explican un $80 \%$ de la variación total. En el caso de la investigación propuesta, a través de tres factores pudo explicarse un $88 \%$ de la variación total, donde se resaltaron las dimensiones capacidad y sostenibilidad y realización de la inversión como las que más influyen dentro de la estructura del desempeño fiscal en el estado de Querétaro.

\section{Conclusiones}

Los análisis realizados previamente demuestran la validez del índice sintético propuesto para evaluar el desempeño fiscal para el estado de Querétaro en el período 2010-2019. En primer lugar, porque los resultados se ajustan a los que muestran otros índices donde se ha aplicado el análisis de componentes principales, y como segundo elemento, el nivel de concordancia que tiene en relación con los indicadores e índices utilizados en la literatura.

Con base en el análisis realizado, puede observarse que el índice sintético adelantado de desempeño fiscal permite sintetizar la información de los indicadores seleccionados con la aplicación del análisis de componentes principales. Cabe resaltar que la construcción y el cálculo del índice sintético permite obtener de forma numérica los resultados en materia de gestión fiscal del estado de Querétaro.

Del mismo modo se obtiene información viable que sirve para dar seguimiento a la gestión realizada por el gobierno con base en los resultados del índice de desempeño fiscal para el período 2010-2019. Con los resultados del índice, el estado de Querétaro cuenta con una herramienta de apoyo en el proceso de toma de 
decisiones y en la definición de estrategias para mejorar el desempeño fiscal. Además, debe tomarse en cuenta que para un correcto proceso de gestión fiscal el primer paso es la evaluación del desempeño fiscal.

Al respecto, se establece la categoría de clasificación del desempeño fiscal, con la cual pueden plantearse perspectivas sobre el manejo de las finanzas públicas y la recaudación fiscal. Según la clasificación obtenida por el estado, pueden proponerse medidas dirigidas a mejorar el desempeño fiscal. Los resultados obtenidos del análisis permiten observa que en el período 2016-2019 el estado tenía un nivel alto de desempeño fiscal, pero el inicio de la pandemia ha repercutido en los diferentes indicadores en esta materia. Lo anterior ha afectado la capacidad financiera del estado como resultado de la paralización de sectores importantes de su economía, como el sector automotriz. La mayor fuente de recursos propios de Querétaro en 2019 fueron las transferencias recibidas por el gobierno federal como consecuencia de la disminución de los ingresos tributarios, lo que afectó el desempeño fiscal.

La metodología aplicada en la presente investigación demuestra la relevancia que tienen los métodos de estadística multivariada en las ciencias económicas administrativas para agrupar y sintetizar un conjunto de variables que permita explicar de forma más simple el desempeño fiscal del estado de Querétaro. Otra ventaja que presenta dicha metodología es la de establecer un criterio de clasifica- ción según el resultado del índice sintético en bajo, medio, alto y muy alto, así como la construcción de indicadores parciales que permitan evaluar las dimensiones del desempeño fiscal.

En el trabajo se cumplió con el objetivo propuesto de construir un índice sintético adelantado de desempeño fiscal para el estado de Querétaro con el que pueda evaluarse de forma sencilla el desempeño fiscal. Además, cabe destacar que con el índice puede realizarse el seguimiento de los resultados integrales del estado y la gestión que desempeña el gobierno en materia fiscal.

Asimismo, con el índice sintético pueden observarse y analizarse los resultados en el tiempo, lo que ayuda en el proceso de gestión y mejora de las actividades de recaudación fiscal y también a proponer el indicador a nivel municipal para conocer el desempeño fiscal de los municipios que componen el estado.

\section{Información complementaria}

Contribuciones de autoría: el autor principal de la presente investigación, Omar Bautista Hernández, realizó la revisión documental, la propuesta de la metodología, la redacción y presentación del artículo de investigación, así como el análisis de los resultados obtenidos.

Conflictos de interés: el autor principal, Omar Bautista Hernández, declara que el presente artículo no presenta conflicto de intereses en relación con la revista y sus editores. 
Financiamiento: la presente investigación fue financiada con los recursos propios del autor, producto de su esfuerzo y dedicación a la investigación en el área de impuesto, como parte de su proceso de formación doctoral.

Material suplementario: no existe material suplementario para el presente artículo de investigación. La sugerencia es que las referencias bibliográficas sean revisadas para la ampliación del tema.

\section{Referencias}

Andalle, L. (2020). Las reg/as de responsabilidad fiscal federal y sus implicaciones en los niveles de gobierno en Argentina 2004-2014 [tesis de doctorado, Universidad de Murcia]. Proyecto de investigación. Digitum: Repositorio Institucional de la Universidad de Murcia. https://bit.ly/3pxl7M1

Ávila Rodríguez, J. A. \& Solano-Pérez, H. (2018). Análisis comparativo del indicador del desempeño fiscal de los departamentos categoría 4 y 1 de Colombia. Vigencia 2016 [trabajo de investigación, Universidad Católica de Colombia]. Repositorio Institucional Universidad Católica de Colombia - RIUCaC. https://bit.ly/3rl99Se

Blancas Peral, F. J., Contreras-Rubio, I. \& Ramírez-Hurtado, J. M. (2006). Construcción de indicadores sintéticos: una aproximación para maximizar la discriminación. Anales de ASEPUMA, 19, 1-23. https://bit.ly/301Mnje

Cano Ortega, N. A. (2019). Análisis comparativo del índice de desempeño fiscal, para los departamentos de Cundinamarca y Chocó. Vigencia 2017 [especialización en Finanzas y Administración Pública, Universidad Militar Nueva
Granada]. Repositorio Institucional UMNG. https://bit.ly/3dA9pKJ

Carvajal, A. \& López-Camargo, O. D. A. J. (2020). El desempeño fiscal municipal y su influencia en el crecimiento económico de los municipios colombianos. Clío América, 14(28), 504-516. https://doi. org/10.21676/23897848.4027

Cochrane, J. H. (2021). A fiscal theory of monetary policy with partially-repaid long-term debt. Review of Economic Dynamics. https://doi.org/10.1016/j. red.2021.06.001

Corrêa da Silva, M., Viana de Souza, F. J., Medeiros Martins, J. D. \& de Barros Câmara, R. P. (2020). Fatores explicativos da gestão fiscal em municípios brasileiros. Revista Contemporânea de Contabilidade, 17(42), 26-37. https:// doi.org/10.5007/2175-8069.2020v17 n42p26

Dorado Corona, F. M., Solís Corvo, M. V. \& Cruz Castañeda, V. (2021). Alternativa metodológica Pos-COVID-19 para la construcción de un indicador sintético adelantado. Economía y Desarrollo, Revista de la Facultad de Economía Universidad de la Habana, 165(Supl. 1), e6. https://bit.ly/3pz3xHh

Duque, I., Domínguez-Berjón, M. F., Cebrecos, A., Prieto-Salceda, M. D., Esnaola, S., Calvo Sánchez, M. \& Marí-Dell'Olmo, M. (2021). Índice de privación en España por sección censal en 2011. Gaceta Sanitaria, 35(2), 113-122. https://doi.org/10.1016/j. gaceta.2019.10.008

Durán Romero, G. (2000). Desarrollo sostenible. Una revisión de los indicadores económicos de sustentabilidad. Investigación Económica, 60(231), 109138. https://bit.ly/3rGq1sN 
El Anshasy, A. A. \& Katsaiti, M. S. (2013). Natural resources and fiscal performance: Does good governance matter? Journal of Macroeconomics, 37, 285-298. https://doi.org/10.1016/j. jmacro.2013.05.006

Eyraud, L. \& Lusinyan, L. (2013). Vertical fiscal imbalances and fiscal performance in advanced economies. Journal of Monetary Economics, 60(5), 571-587. https://doi.org/10.1016/j. jmoneco.2013.04.012

Flores Araujo, A. D., \& Martínez Gómez, F. (2019). La baja recaudación fiscal en México. Equilibrio Económico, Revista de Economía, Política y Sociedad, 15 (2) (48), 148-175. https://bit.ly/3rJBZBZ

Gómez Giraldo, A. C., Ortíz Ocampo, M. J. \& Rodríguez Oviedo, G. (2013). Medición y evaluación de desempeño fiscal a través del indicador sintético, en el marco de la NGP: AMCO, 2010 [Universidad Católica de Pereira]. Repositorio Institucional de la Universidad Católica de Pereira - RIBUC. https://bit.ly/3GILgnz

Hernandez Garcia, E. A., Zápata Prado, M. A., González Tique, S. L. \& Armas Moyano, A. A. (2020). Restricción presupuestaria y desempeño fiscal de Santander de Quilichao-Colombia, 2004-2016. Revista GEON (Gestión, Organizaciones y Negocios), 7(1), 179-204. https://doi. org/10.22579/23463910.201

Hernández Ocampo, J. E. (2017). Dinámicas y perspectivas del indice del desempeño fiscal de Cali: un ejercicio de dinámica de sistemas para el periodo 2016-2026 [tesis de grado, Universidad del Valle]. Biblioteca digital Universidad del Valle. https://bit.ly/3y7rUQu

Huerta Villalobos, M. M., Alcantar López, C. O. \& Flores Ortega, G. (2021). Los riesgos fiscales en la política econó- mica de México. Diagnóstico Fácil empresarial, 11(Enero-Junio 2019/año 6), 5-11. https://doi.org/10.32870/ dfe.vi11\%20Enero-Junio\%202019/ año\%206.40

Ibarra Salazar, J., González, H. \& Sotres Cervantes, L. (2012). Aspectos políticos de la dependencia financiera en los municipios mexicanos. Revista Mexicana de Ciencias Políticas y Sociales, 58(217). https://doi.org/10.1016/ s0185-1918(13)72278-5

Instituto Nacional de Estadística, Geografía e Informática - INEGI. (2020). Anuario estadístico: Querétaro: Instituto Nacional de Estadística, Geografía e Informática. https://www.inegi.org.mx/

Lacaze, M. V., Alegre, P., Errea, D., Atucha, A. J., Volpato, G., Blanco, G., Fernández, M. \& Bianchetti, L. (2021). Indicador sintético de actividad económica de General Pueyrredon: avances en su construcción. II Congreso Internacional de DesarroIlo Teritorial. https://bit.ly/31GBflP

Lami, E., Imami, D., Pugh, G. \& Hashi, I. (2021). Fiscal performance and elections in the context of a transition economy. Economic Systems, 45(2), 100886. https://doi.org/10.1016/j. ecosys.2021.100886

León González, Á., Llinás Solano, H. \& Tilano, J. (2008). Análisis multivariado aplicando componentes principales al caso de los desplazados. Ingeniería y Desarrollo, (23), 119-142. https://bit. ly/3DsmZKZ

López Martín, B., Ramírez de Aguilar, A. \& Sámano Peñaloza, D. (2020). Consideraciones sobre política fiscal y expectativas de inflación en México. Investigación Económica, 79(312), 63-88 https://doi.org/10.22201/ fe.01851667p.2020.312.75372 
López Pérez, S. J. \& Vence, X. (2021). Estructura y evolución de ingresos tributarios y beneficios fiscales en México. Análisis del periodo 1990-2019 y evaluación de la reforma fiscal de 2014. El Trimestre Económico, 88(350), 373417. https://doi.org/10.20430/ete. v88i350.1104

Llamas Rembao, L. I., Huesca Reynoso, L. \& Gutiérrez Flores, L. (2020). Abordajes metodológicos y empíricos de la progresividad tributaria: una aplicación para el sistema fiscal de México. Economía: Teoría y Práctica, (53), 121-149. https://doi.org/10.24275/ etypuam/ne/532020/llamas

Madrigal-Delgado, G. J., Camacho-Castro, C., González-Franco, R. A. \& Bueno-Cevada, L. E. (2018). Desempeño fiscal municipal del estado de Sinaloa: Propuesta de índice sintético para evaluaciones fiscales. Economía, Sociedad y Territorio, 18(57), 359-396. https://doi. org/10.22136/est20181175

Madrigal Delgado, G. J. \& Bueno Cevada, L. E. (2019). Transferencias, Gestión Fiscal Municipal y la Desigualdad Interregional en México. Investigación Administrativa, 48(124). https://bit. ly/3Ezy3ah

Malvicino, F., Pereira, M. \& Trajtenberg, L. (2020). Índices de actividad económica provincial en base a un modelo factorial dinámico. Argentina 1997-2019. Cuadernos Del CIMBAGE, 2(22), 69-100. https://bit.ly/3IF1X5]

Mendoza Velázquez, A. (2010). Indicadores de desempeño, presión y vulnerabilidad de las finanzas públicas estatales en México. El Trimestre Económico, 77(307), 603-647. https://bit.ly/307z7Du

Mendoza Velázquez, A. \& Rubio García, M. (2020). Entorno fiscal federal y eficiencia de los gobiernos locales en México: el impacto de la crisis de 2008. Economía: Teoría y Práctica, (53), 151-176. https://doi.org/10.24275/ etypuam/ne/532020/mendoza

Molinos-Senante, M., Delgado-Antequera, L. \& Gómez, T. (2021). Measuring the quality of service of water companies: A two-stage goal programming synthetic index proposal. Socio-Economic Planning Sciences, 101140. https:// doi.org/10.1016/j.seps.2021.101140

Mondéjar-Jiménez, J., Mondéjar-jiménez, J. A. \& Vargas-Vargas, M. (2009). Medición de la evolución económica del sector agrario mediante indicadores sintéticos en Castilla-La Mancha. Agrociencia, 43(3), 309-318. https://bit. ly/3y6p120

Mondéjar-Jiménez, J. \& Vargas-Vargas, M. (2008). Indicadores sintéticos: una revisión de los métodos de agregación. Economía, Sociedad y Territorio, 8(27), 565-585. https://bit.ly/3dtKQ22

Montenegro, D. I. (2021). Diseño de un modelo para el dinamismo empresarial: personas, innovación y tecnología como elementos críticos. $3 C$ Empresa. Investigación y Pensamiento Crítico, 10(3), 51-85. https://bit.ly/3pz8nEB

Montiel-Morales, G., Hernandez Contreras, R. G., Tenorio Martínez, J. F. \& Machuca-Rodríguez, D. L. (2020). Análisis de la Capacidad Contributiva en México 2020. Revista Global de Negocios, 8(1), 73-87. https://bit.ly/3ItTagy

Morales, V. \& Bayona, S. (2020). Modelo conceptual de factores y su influencia en los niveles de desarrollo del e-gobierno municipal. Revista Ibérica de Sistemas e Tecnologias de Informação, 28-41. https://bit.ly/3rPeHui 
Moreno-Brid, J. C., Pérez Benítez, N., Villarreal Páez, H. J. \& Salat, I. (2019). Retos de política fiscal para el desarrollo. Economía UNAM, 16(46), 61-72. https://bit.ly/3rJ7Vq3

Navarro, D., Vallejo, I. \& Navarro, M. (2020). Análisis de la vulnerabilidad social a los riesgos naturales mediante técnicas estadísticas multivariantes. Investigaciones Geográficas, (74), 29-49. https://doi.org/10.14198/INGEO2020.NVN

Pliego Quintana, M. (2014). Análisis coyuntural de la política fiscal en México 1993-2004. Economía UNAM, 11(32), 59-76. https://doi.org/10.1016/ S1665-952X(14)70452-9

Ramírez Rodríguez, R., Erquizio Espinal, A., Díaz Carreño, M. A. \& Terrones Cordero, A. (2017). Capacidad, productividad y desempeño fiscal en las entidades federativas de México, 2010-2014. Paradigma Económico, 9(2), 93-126. https://bit.ly/3rHJXLO

Rao, S. H. (2021). Transportation synthetic sustainability indices: A case of Taiwan intercity railway transport. Ecological Indicators, 127, 107753. https://doi. org/10.1016/j.ecolind.2021.107753

Reza-Paocarina, E. B., Londoño-Espinosa, S., Enríquez-Fuenmayor, J. E. \& León-Serrano, L. A. (2021). Aplicación metodológica de riesgos fiscales de corto plazo. Dominio de las Ciencias, 7(2). http://dx.doi.org/10.23857/dc. v7i2.1801

Sánchez, M. D. P., Lozano, D. \& Moreno, M. (2016). Índice de Desempeño Integral ajustado a las localidades de Bogotá DC 1. Revista de Administração Pública, 50, 611-634. https://doi. org/10.1590/0034-7612141819
Sánchez León, O. L. (2021). El papel del presupuesto público en el bienestar social de una nación. InterNaciones, (20), 137-151. https://doi. org/10.32870/in.vi20.7176

Sánchez Sanabria, Y. K. (2009). Transparencia presupuestal y desempeño fiscal: un análisis de su relación para el caso de los gobiernos generales del nivel nacional en los países de la OCDE [tesis de maestría, Universidad Nacional de Colombia]. Repositorio Institucional UN. https://bit.ly/3DzElzz

Sánchez-Torres, F. (2008). La descentralización en Colombia: el desempeño fiscal de las entidades subnacionales. Inter-American Development Bank. https://bit.ly/3pERJTW

Silva-Treviño, J. G., Macías-Hernández, B. A., Tello-Leal, E. \& Delgado-Rivas, J. G. (2021). La relación entre la calidad en el servicio, satisfacción del cliente y lealtad del cliente: un estudio de caso de una empresa comercial en México. CienciaUAT, 15(2), 85-101 https://doi. org/10.29059/cienciauat.v15i2.1369

Tejero, J., Fernández, J. \& Mira, J. (2011). Validez y fiabilidad de un indicador sintético de calidad de la atención hospitalaria. Revista de Calidad Asistencial, 26(4), 234-241. https://doi.org/10.1016/j.cali.2011.02.003

Tinto Arandes, J., Guijarro Cordero, A. L. \& Molina T., M. E. (2021). Método multivalente en la construcción de indicadores para el desarrollo sostenible. Teorías, Enfoques y Aplicaciones en las Ciencias Sociales, 13(28), 20-30. https://bit.ly/3rPhB2a

Urrea, A. F., Galindo, J. L., Osorio, M. V., Jimenez, D. R. \& Salas, L. E. (2020). Nueva Medición de Desempeño Fiscal 
Territorial: Departamento Nacional de Planeación. https://bit.ly/3rLGo7e

Valencia, A. R., Reynoso, L. H., Castro, M. C. (2015). Incidencia del Sistema Fiscal en México 2002-2012. Economía Informa, 390, 3-27. Doi: https://doi. org/10.1016/S0185-0849(15)30002-5

Valenzuela-Reynaga, R, \& Hinojosa-Cruz, A. V. (2017). Las transferencias federales, los contrapesos políticos y los ingresos fiscales estatales en México. Economía UNAM, 14(42), 4771. Doi: https://doi.org/10.1016/j. eunam.2017.09.002

Vásquez, M., Camardiel, A. \& Ramírez, G. (2000). Una propuesta para la construcción de un índice sintético de pobreza. Revista Venezolana de Análisis de Coyuntura, 6(1), 121-142. https://bit. ly/3IE7LX4

Wang, K. H., Liu, L., Adebayo, T. S., Lobon, O. R., \& Claudia, M. N. (2021). Fiscal decentralization, political stability and resources curse hypothesis: A case of fiscal decentralized economies. Resources Policy, 72, 102071. https://doi.org/10.1016/j.resourpol.2021.102071
Zhang, D. \& Hussain, H. I. (2021). Nexus between fiscal imbalance and emissions reduction: New evidence from developing economies. Journal of Environmental Management, 297, 113360. https://doi.org/10.1016/j. jenvman.2021.113360

\section{Biodata autor}

Omar Bautista Hernández. Contador Público, Universidad Autónoma de Querétaro. Maestro en Impuesto, Universidad Autónoma de Querétaro. Candidato a doctor en Administración, Universidad Autónoma de Querétaro. Secretario académico y profesor titular de las materias Teoría General de la Tributación, Impuestos Directos II y Código Fiscal de la Federación, Facultad de Contaduría y Administración de la Universidad Autónoma de Querétaro. Maestro asesor del equipo de fiscal en los maratones nacionales de ANFECA, logrando el tercer puesto a nivel nacional representando a la FCA UAQ. Intereses de investigación: impuestos, desempeño fiscal. Política fiscal, desarrollo económico, código fiscal. ORCID: https:// orcid.org/0000-0003-0027-0898 